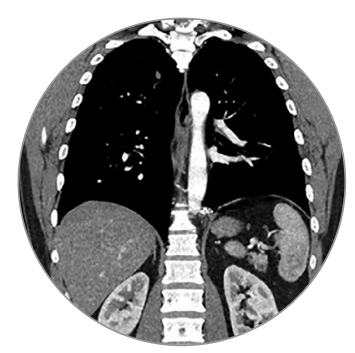

\title{
Neumonía recurrente como manifestación de secuestro pulmonar. Presentación de caso
}

\author{
Recurrent Pneumonia as a Manifestation of Pulmonary Sequestration. \\ Case Report
}

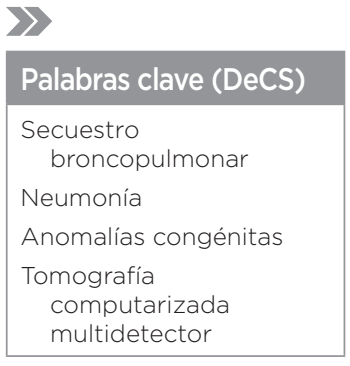

Key words (MeSH)

Bronchopulmonary sequestration

Pneumonia

Congenital anomalies

Multidetector computed tomography
Médica residente de Radiología e Imágenes Diagnósticas. Universidad Autónoma de Bucaramanga. Bucaramanga, Colombia.

2Estudiante de Medicina, Universidad Autónoma de Bucaramanga. Bucaramanga, Colombia.

${ }^{3}$ Estudiante de Medicina, Universidad Industrial de Santander. Bucaramanga, Colombia.

${ }^{4}$ Radiólogo. Departamento de Radiología, Clínica Carlos Ardila Lülle. Bucaramanga, Colombia.

${ }^{5}$ Radiólogo. Departamento de Radiología, Clínica Carlos Ardila Lülle. Bucaramanga, Colombia.

Departamento de Radiología e Imágenes Diagnósticas Fundación Oftalmológica de Santander, Clínica Carlos Ardila Lülle. Bucaramanga, Colombia.

\section{Case Report}

Milena Rey Ayala Erick Daniel Villarreal Ibáñez ${ }^{2}$ Silvia Nathalia Vera Campos ${ }^{3}$

Carlos Alfonso Rey Graz ${ }^{4}$

Federico Guillermo Lubinus Badillo ${ }^{5}$

\section{Resumen}

El secuestro pulmonar es una enfermedad congénita del tracto respiratorio que compromete el parénquima pulmonar y su vasculatura, puede dividirse en intralobar o extralobar dependiendo de su relación con la pleura visceral normal. El subtipo extralobar usualmente se diagnostica prenatalmente o en los primeros meses de vida, mientras que el intralobar se presenta en adultos jóvenes. Representa aproximadamente el 0,15-6,4 \% de todas las malformaciones pulmonares congénitas y no tiene diferencias respecto al sexo con una relación 1:1. Se presenta el caso de un paciente masculino adulto medio con neumonías a repetición en quien se realizan estudios imagenológicos con hallazgos tomográficos compatibles con secuestro pulmonar intralobar en el segmento basal posterior izquierdo, con el objetivo de realizar una revisión de la literatura para plantear una discusión sobre la importancia del diagnóstico adecuado y a tiempo de esta patología. Es necesario tener una alta sospecha clínica - se trata de una enfermedad de presentación variable-, para solicitar el estudio imagenológico más adecuado, puesto que las imágenes son las que dan el diagnóstico definitivo del secuestro pulmonar. En algunos casos, el manejo se puede realizar de manera mínimamente invasiva a cargo de radiología intervencionista, como en este caso.

\section{Summary}

Pulmonary sequestration is a congenital respiratory disease that involves the pulmonary parenchyma and its vasculature. It can be divided into intralobar or extralobar depending on its relationship with normal visceral pleura. The extralobar subtype is usually diagnosed prenatally or in the first months of life, while the intralobar occurs in young adults. It represents approximately 0.15-6.4\% of all congenital lung malformations and does not show differences with respect to sex with a 1:1 ratio. We present the case of an average adult male patient with recurrent pneumonia in whom multiples imaging studies are performed, with tomographic findings compatible with intralobar pulmonary sequestration in the left posterior basal segment, with the aim of performing a literature review to raise a discussion on the importance of the proper and early diagnosis of this pathology. It is necessary to have a high clinical suspicion -it is a disease of variable presentation-, to request the most appropriate imaging study, since the images are those that give the definitive diagnosis of pulmonary sequestration. In some cases, management can be performed in a minimally invasive manner by interventional radiology, as in this case.

\section{Introducción}

El secuestro pulmonar (SP) es una enfermedad congénita del tracto respiratorio caracterizada por un área de tejido pulmonar displásico y no funcional, el cual no está en continuidad con el árbol traqueobronquial y además se encuentra irrigado por vasos sistémicos aberrantes, generalmente provenientes de la aorta (1). Sin embargo, estos vasos también pueden originarse del tronco celíaco, la arteria esplénica, las arterias intercostales e incluso de las arterias coronarias (2).
Representa aproximadamente el 0,15-6,4\% de todas las malformaciones pulmonares congénitas y no muestra diferencias con respecto al sexo, con una relación 1:1 $(3,4)$. Se han descrito dos tipos de secuestro pulmonar: intralobar (SPI) y extralobar (SPE). La prueba diagnóstica de referencia es la arteriografía. Se trata del caso clínico de un paciente adulto medio con diagnóstico de secuestro pulmonar intralobar y quien recibió manejo mínimamente invasivo con resultados satisfactorios. Se realiza un análisis del caso y revisión de la literatura. 


\section{Caso clínico}

Paciente masculino adulto medio con antecedente de neumonías a repetición, que acude al servicio de urgencias por cuadro febril y síntomas respiratorios. Al ingreso se registra temperatura de $38,3{ }^{\circ} \mathrm{C}$, taquicárdico y murmullo vesicular disminuido en la base pulmonar izquierda. Se realizan paraclínicos, los cuales reportan leucocitosis de $16.880 \mathrm{~mm}^{3}$, con neutrófilos de $85 \%$ y PCR de $101,48 \mathrm{mg} / \mathrm{L}$. Se realiza radiografía de tórax PA y lateral con evidencia de opacidad de tipo alveolar a la altura del lóbulo inferior izquierdo en los segmentos inferior y posterior (figura 1). Se inicia antibioticoterapia para manejo del cuadro agudo; sin embargo, por el antecedente de neumonías a repetición se procede a realizar tomografía computarizada (TC) de tórax con medio de contraste, en la cual se observan infiltrados alveolares en el segmento basal posterior del lóbulo inferior izquierdo, con estructura vascular arterial que se origina de la aorta torácica distal, con drenaje venoso a las venas pulmonares, hallazgos compatibles con secuestro pulmonar intralobar en el segmento basal posterior izquierdo (figura 2). Ante los hallazgos descritos por imagenología y la clínica reiterativa de procesos infecciosos pulmonares se le realiza embolización y oclusión selectiva de vaso aberrante arterial mediante espirales (coils) y micropartículas, procedimiento realizado sin complicaciones, con evolución satisfactoria y adecuado control postoperatorio (figuras 3 y 4 ).

\section{Discusión}

El secuestro pulmonar intralobar corresponde al $75 \%$ de todos los secuestros pulmonares. Este consiste en un segmento pulmonar anómalo que comparte pleura visceral con un lóbulo pulmonar normal y carece de una comunicación normal con el árbol traqueobronquial, irrigado principalmente por ramas anómalas provenientes de la aorta, las cuales se localizan generalmente a través del ligamento pulmonar inferior y que drenan a través de las venas pulmonares al atrio izquierdo. Su localización principalmente se da en el lóbulo inferior con un predominio ligeramente mayor en el pulmón izquierdo que en el derecho (3). Hallazgo concordante con el caso presentado en el que se evidenciaron características clínicas e imagenológicas en el campo pulmonar izquierdo.

Ambos tipos de secuestro pulmonar presentan una patogénesis distinta. El SPE es una anomalía congénita cuya etiopatogenia aún es un poco controvertida, pero se cree que se desarrolla a partir de un brote pulmonar supernumerario que obtiene su irrigación sanguínea a partir de los vasos esplácnicos primitivos que rodean el intestino anterior (5).

Por otro lado, el SPI no se encuentra relacionado con malformaciones congénitas, por lo cual se piensa que es una condición adquirida secundaria a múltiples infecciones, procesos inflamatorios crónicos pulmonares o cuadros obstructivos bronquiales (6). Esto es consistente con el diagnóstico del paciente, en quien el único antecedente de importancia era infección del tracto respiratorio inferior tipo neumonía a repetición, el cual es uno de los síntomas más comunes de pacientes con secuestro pulmonar intralobar. El diagnóstico del SPI se puede realizar a cualquier edad en más de la mitad de los pacientes. Generalmente se diagnostica después de la segunda década de la vida. Por el contrario, el SPE se diagnostica principalmente durante el periodo fetal o neonatal asociado a hernias diafragmáticas, anomalías cardiacas e incluso hipoplasia pulmonar $(3,7)$.

Las características clínicas de los pacientes que tienen SPI se presentan típicamente con una historia crónica de tos, expectoración mucopurulenta y neumonías recurrentes, generalmente causadas por bacterias piógenas (8).

Cuando se sospecha un SP los exámenes diagnósticos deben enfocarse hacia la identificación de circulación aberrante y la búsqueda de comunicaciones con el tracto gastrointestinal o anomalías congénitas asociadas (6). El examen inicial debe ser la radiografía simple de tórax, en el que se pueden identificar anormalidades parenquimatosas o sombras vasculares que sugieran el diagnóstico. En este caso, los hallazgos radiográficos fueron consistentes con neumonías izquierdas en múltiples ocasiones; sin embargo, son hallazgos inespecíficos para el diagnóstico de secuestro pulmonar.

La angiografía se considera el examen de referencia para identificar la circulación nutricia del SP; sin embargo, aunque este estudio es capaz de identificar el suministro arterial aberrante de un pulmón secuestrado, es invasivo, requiere hospitalización y no proporciona suficiente información sobre la estructura pulmonar. En la actualidad, existen técnicas de imagen no invasivas que incluyen la tomografía computarizada (TC), la resonancia magnética (RM) y la ecografía Doppler con medio de contraste que han desplazado la angiografía diagnóstica como primera opción.

En particular, se ha demostrado que la TC multidetector con medio de contraste endovenoso es igualmente efectiva que la angiografía y si estas dos se realizan simultáneamente sirven para confirmar un suministro arterial anómalo y para distinguir secuestro pulmonar de otras opacidades pulmonares (6).

La TC es muy útil en la identificación de los cambios parenquimatosos y la extensión del compromiso pulmonar, los hallazgos radiológicos típicos del secuestro pulmonar intralobar son una masa homogénea o heterogénea de tejido blando en el lóbulo inferior, usualmente acompañado de zonas de consolidación heterogénea y cambios enfisematosos en los bordes de la lesión. Por lo general, el resto del parénquima pulmonar no secuestrado se encuentra sano, hallazgos consistentes con los descritos en el caso, en donde se encontraron alteraciones parenquimatosas a la altura del segmento basal posterior izquierdo asociado a un vaso arterial proveniente de circulación sistémica (aorta) en el TC de tórax simple y con medio de contraste.

El tratamiento usualmente es quirúrgico, incluso algunos autores lo consideran hasta en los casos asintomáticos, principalmente debido a la transformación maligna del parénquima aberrante y otras posibles complicaciones (9). Se considera la resección total del parénquima pulmonar secuestrado por videotoracoscopia como el manejo quirúrgico de elección (10). Sin embargo, algunos autores consideran la embolización arterial como una alternativa que permite la reducción del tamaño de la lesión, para una eventual extirpación posterior. Un suministro arterial sistémico anormal no solo confirma el diagnóstico, sino que también proporciona una hoja de ruta vascular preoperatoria para el cirujano, por lo tanto, minimiza las posibilidades de lesiones vasculares involuntarias (7). En el caso presentado se realizó una embolización endovascular del vaso arterial aberrante a cargo de radiología intervencionista. Mediante arteriografía posterior al procedimiento se confirmó la devascularización total del árbol arterial correspondiente al secuestro pulmonar. El procedimiento se llevó a cabo sin complicaciones y el paciente no ha presentado nuevos episodios respiratorios bajos agudos, lo cual confirma el SP como predisponente para el desarrollo de neumonía a repetición. Se realizó un control imagenológico dos meses después del procedimiento intervencionista que evidenció una evolución satisfactoria. 

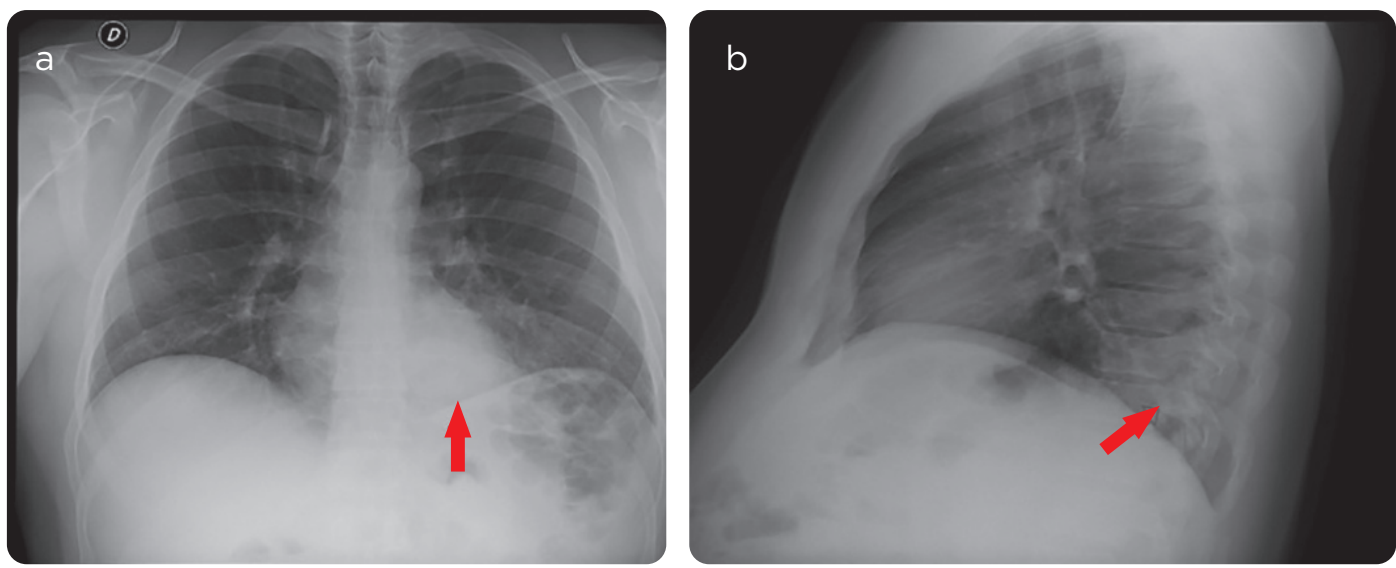

Figura 1. Radiografía de tórax PA y lateral, que evidencia opacidad de tipo alveolar a nivel de lóbulo inferior izquierdo (flechas).
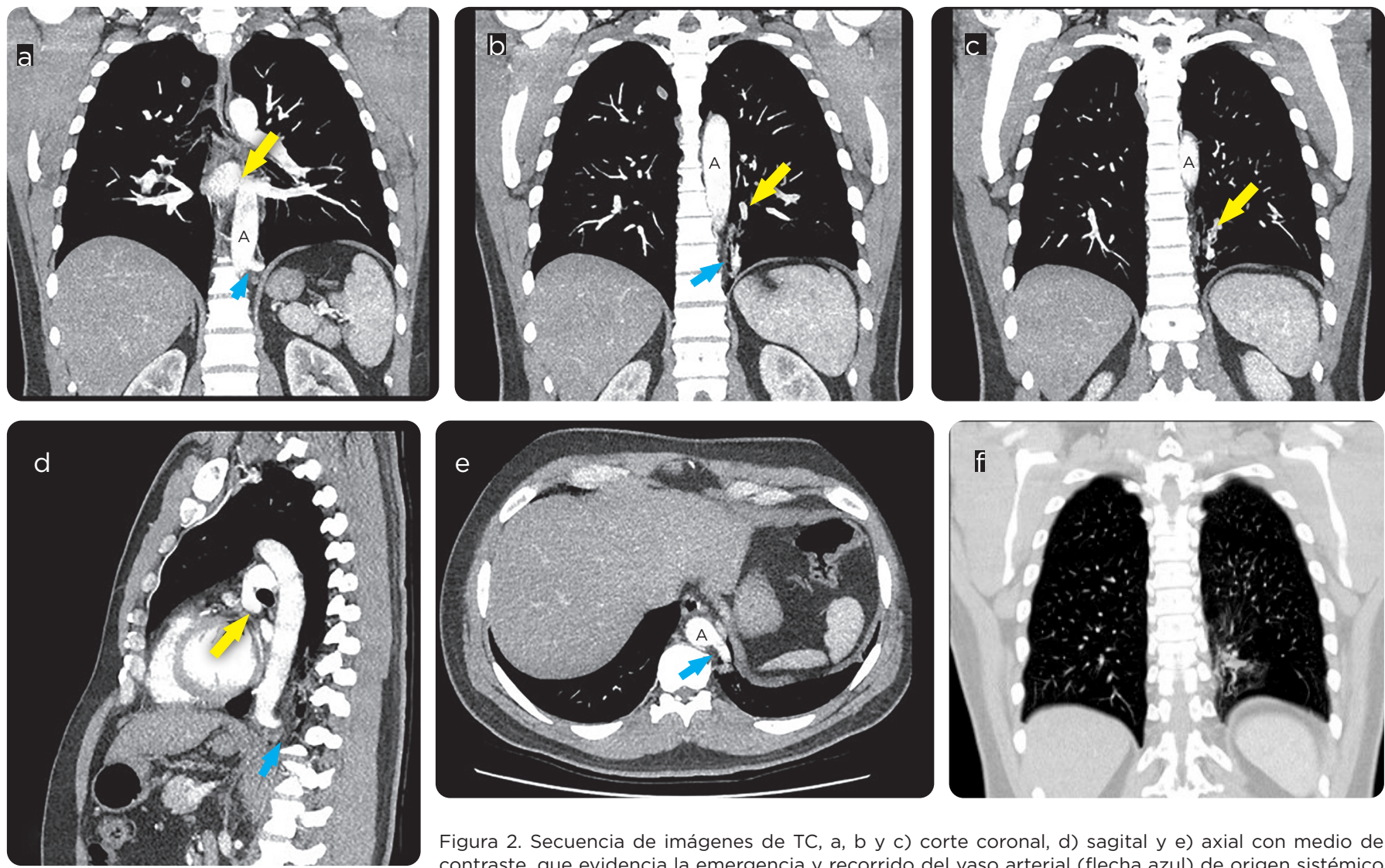

Figura 2. Secuencia de imágenes de TC, a, b y c) corte coronal, d) sagital y e) axial con medio de contraste, que evidencia la emergencia y recorrido del vaso arterial (flecha azul) de origen sistémico (A: Aorta torácica) y el drenaje venoso a (flecha amarilla) venas pulmonares. f) Imagen en corte coronal ventana para pulmón que demuestra infiltrados de tipo alveolar en el parénquima.
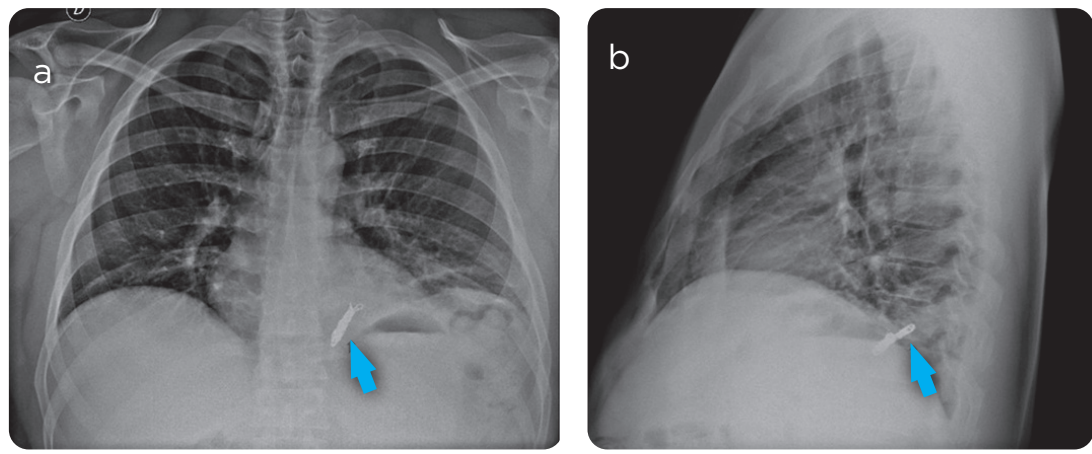

Figura 3. Radiografía de tórax PA y lateral en la cual se observa material de embolizacion en base pulmonar izquierda, sin alteraciones significativas en campos pulmonares. 

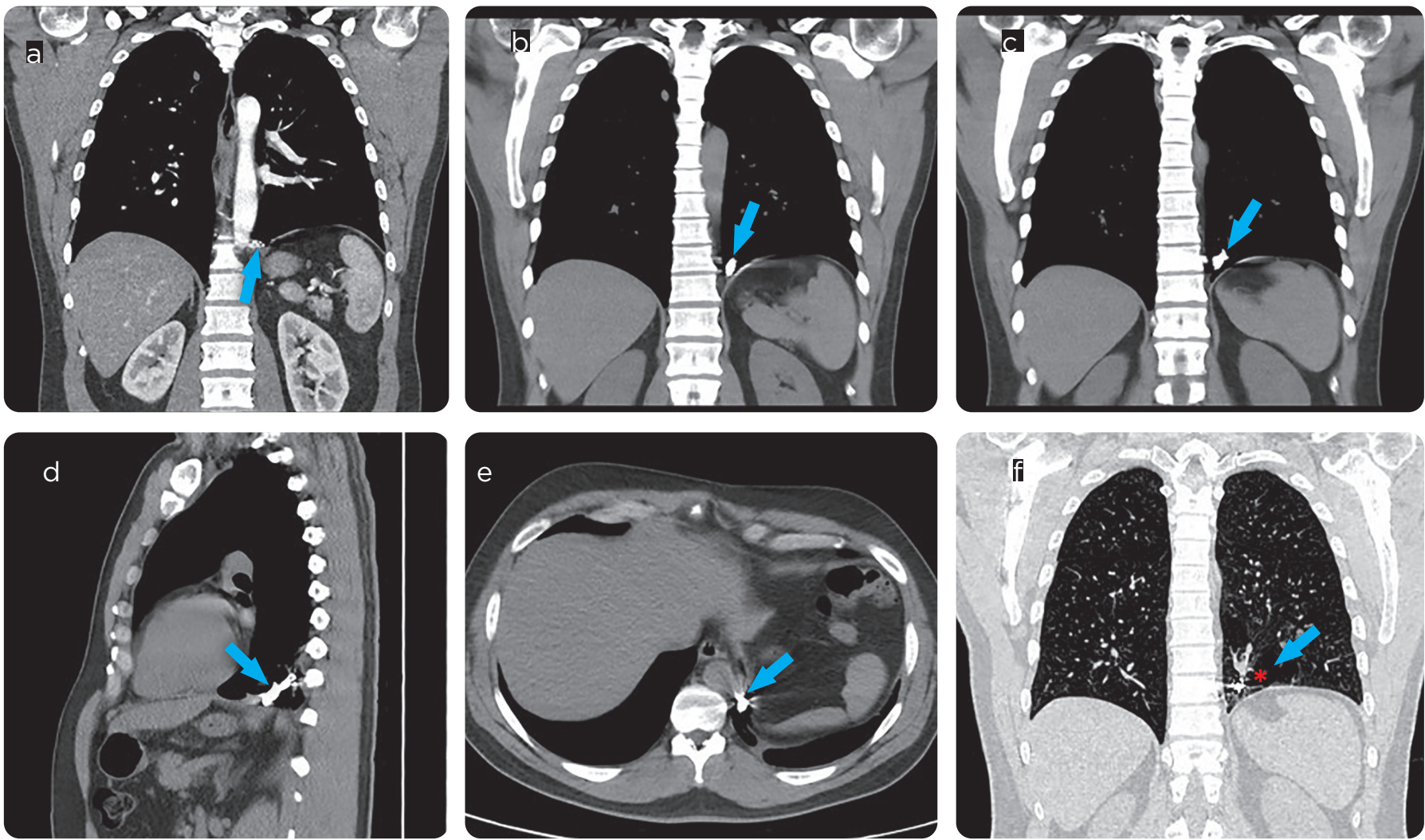

Figura 4. Secuencia de imágenes de TC de tórax en cortes a, b, y c) coronales, d) sagital y e) axial, en cortes similares a la figura 2 (comparativamente pretratamiento y postratamiento), que evidencian material quirúrgico de embolización (flecha azul) en vaso arterial aberrante y f) corte coronal en ventana para pulmón, que demuestra tractos fibrosos y pérdida del volumen del segmento comprometido (asterisco). Cambios normales esperados para el procedimiento.

\section{Conclusiones}

El secuestro pulmonar es una patología congénita poco frecuente que se presenta prenatalmente $\mathrm{y} / \mathrm{o}$ en el periodo neonatal (subtipo extralobar) y en los adultos jóvenes (subtipo intralobar), que generalmente cursa con infecciones del tracto respiratorio inferior a repetición sin otros hallazgos característicos. La arteriografía se considera el método estándar para el diagnóstico, pero en la actualidad se acepta la angiotomografía de tórax como método de elección para hacer evidente el vaso arterial aberrante asociado a los cambios parenquimatosos descritos, lo que da a entender que el diagnóstico de esta patología es netamente radiológico y de ahí la importancia de que los radiólogos estén familiarizados con este tipo de enfermedades, con el fin de evitar errores diagnósticos y demoras en el tratamiento. Como se evidenció, en casos seleccionados se puede realizar por medio de embolización, lo que ofrece una alternativa terapéutica menos invasiva que la resección convencional del tejido pulmonar displásico.

\section{Referencias}

1. Correia-Pinto J, Gonzaga S, Huang Y, Rottier R. Congenital lung lesions - underlying molecular mechanisms. Semin Pediatr Surg. 2010;19(3):171-9.

2. Newman B. Congenital bronchopulmonary foregut malformations: concepts and controversies. Pediatr Radiol. 2006;36(8):773-91.

3. Savic B, Birtel FJ, Tholen W, Funke HD, Knoche R. Lung sequestration: report of seven cases and review of 540 published cases. Thorax. 1979;34:96-101.

4. Stocker JT, Kagan-Hallet K. Extralobar pulmonary sequestration: analysis of 15 cases. Am J Clin Pathol. 1979;72(6):917-25.
5. Rosado de Christenson ML, Frazier AA, Stocker JT, Templeton PA. Extralobar sequestration: radiologic-pathologic correlation. Radiographics. 1993;13:425-41.

6. Long Q, Zha Y, Yang Z. Evaluation of pulmonary sequestration with multidetector computed tomography angiography in a select cohort of patients: A retrospective study. Clinics (Sao Paulo). 2016;71:392-8.

7. Felker RE, Tonkin IL. Imaging of pulmonary sequestration. AJR Am J Roentgenol 1990;154(2):241-9.

8. Stocker JT, Malczak HT. A study of pulmonary ligament arteries: relationship to intralobar pulmonary sequestration. Chest. 1984;86:611-5.

9. Laberge JM, Bratu I, Flageole H. The management of asymptomatic congenital lung malformations. Paediatr Respir Rev. 2004;5:305-12.

10. Belchis D, Cowan M, Mortman M, Rezvani B. Adenocarcinoma arising in an ex- tralobar sequestration: a case report and review of the literatura. Lung Cancer. 2014;84:92-5.

\section{Correspondencia}

Milena Rey Ayala

Calle Real 5-24

Chico Real I

mrey801@unab.edu.co

Recibido para evaluación: 10 de enero de 2020

Aceptado para publicación: 20 de febrero de 2020 